\title{
Optical forces between dielectric nanoparticles in an optical vortex
}

\author{
David S. Bradshaw and David L. Andrews* \\ Nanostructures and Photomolecular Systems, School of Chemical Sciences, \\ University of East Anglia, Norwich, NR4 7TJ, U.K.
}

\begin{abstract}
We report a study on the optical forces between a pair of dielectric particles, based on quantum electrodynamics. At a fundamental level these forces result from a stimulated scattering process which entails a virtual photon relay between the two particles. Results for a variety of systems are secured from a completely general analysis that accommodates a system with arbitrary dielectric properties (with regard to shape, frequency response etc.) in an optical field of arbitrary complexity. Specific results are obtained and exhibited for: (a) optical forces between nanoparticles, and specifically between carbon nanotubes; (b) the effects of optical ordering, clustering and trapping associated with twisted (LaguerreGaussian) laser beams.
\end{abstract}

Keywords: Optical forces, optical vortices, twisted beams, Laguerre-Gaussian, quantum electrodynamics, nanoparticles, carbon nanotubes, optical tweezers

\section{INTRODUCTION}

The experimental detection of forces on neutral particles due to the radiation pressure of applied optical fields, first recognized by Ashkin in 1970, led to an avalanche of applications. ${ }^{1}$ In particular this discovery led in 1986 to the invention of optical tweezers ${ }^{2}-$ a technique that has become a mainstream tool for the optical trapping ${ }^{3,4}$ and manipulation $^{5}$ of a diverse range of particles, principally in biological and nanotechnological disciplines and ranging from individual atoms to single living cells. In a separate line of development, ${ }^{6}$ it was verified that significant optomechanical forces can be induced between particles through the application of an optical field. Differing methods of analysis have been utilized to derive this force using classical theory ${ }^{7,8}$ and, recently, optical binding between a pair of dielectric particles has been observed. ${ }^{9}$ In this paper we use a molecular quantum electrodynamical (QED) approach to determine results for an optically-induced force between dielectric nanoparticles. A general expression is first derived (section 2A), followed by a detailed analysis of two systems comprising single-walled carbon nanotubes $(\text { SWCNTs })^{10}$ - materials which are currently the subject of intense research due to their unique nanostructures and remarkable combination of conductive, steric and mechanical characteristics. ${ }^{11}$ The two systems that are analysed in detail differ in the angular disposition of the nanotubes with respect to the incoming laser light so that: (i) both SWCNTs are disposed at a variable angle to the electric field vector of the incident light (section 2B) and; (ii) the nanotubes have variable mutual orientation, isotropically distributed with respect to the field vector (section 2C). Employing results from other recently developed theory, which delineates a quantum electrodynamical (QED) formulation for the interaction of Laguerre-Gaussian or other 'twisted' optical beams with matter, ${ }^{12}$ an expression for the torque between a pair of molecules under this radiation type is determined (section 3 ). The results of calculations are performed for chemically identical neutral molecules, and the paper concludes with a discussion.

\section{OPTICALLY-INDUCED FORCE EXPRESSIONS}

\section{A. General result}

The framework of molecular $\mathrm{QED}^{13}$ - in which both radiation and matter are treated quantum mechanically - provides a highly satisfactory theory for the study of optically-induced interactions. Using this approach in multipolar form, the full Hamiltonian, $H$, for a systems of material particles is given by;

$$
H=\sum_{\xi} H_{\mathrm{mol}}(\xi)+\sum_{\xi} H_{\mathrm{int}}(\xi)+H_{\mathrm{rad}},
$$


where $H_{\text {mol }}^{\xi}$ is the Hamiltonian for particle $\xi$ and $H_{\text {rad }}$ denotes the radiation field. The Hamiltonian $H_{\text {int }}^{\xi}$ represents the interaction of the field with $\xi$ and, within the electric dipole, is given by;

$$
H_{\mathrm{int}}^{\xi}=-\sum_{\xi} \mu(\xi) \cdot \mathbf{d}^{\perp}\left(\mathbf{R}_{\xi}\right)
$$

with $\mu(\xi)$ and $\mathbf{R}_{\xi}$ as the electric-dipole moment operator and the position vector, respectively. The operator $\mathbf{d}^{\perp}\left(\mathbf{R}_{\xi}\right)$ represents the transverse electric displacement field and is often presented in a plane wave mode expansion involving summations over all wave-vectors, $\mathbf{p}$, and polarisations, $\lambda-$ this is expressed as;

$$
\mathbf{d}^{\perp}\left(\mathbf{R}_{\xi}\right)=\mathrm{i} \sum_{\mathbf{p}, \lambda}\left(\frac{\hbar c p \varepsilon_{0}}{2 V}\right)^{1 / 2}\left\{\mathbf{e}^{(\lambda)}(\mathbf{p}) a^{(\lambda)}(\mathbf{p}) e^{\mathrm{i}\left(\mathbf{p} \cdot \mathbf{R}_{\xi}\right)}-\overline{\mathbf{e}}^{(\lambda)}(\mathbf{p}) a^{\dagger(\lambda)}(\mathbf{p}) e^{-\mathrm{i}\left(\mathbf{p} \cdot \mathbf{R}_{\xi}\right)}\right\} .
$$

Here $\mathbf{e}^{(\lambda)}(\mathbf{p})$ is the polarisation unit vector $\left(\overline{\mathbf{e}}^{(\lambda)}(\mathbf{p})\right.$ being its complex conjugate), $V$ is an arbitrary quantisation volume and $a^{(\lambda)}(\mathbf{p}), a^{\dagger(\lambda)}(\mathbf{p})$ are respectively the photon annihilation and creation operators for a mode (p, $\left.\lambda\right)$. A general result for a laser-induced force between a pair of neutral species A and B can be determined via an expression for the energy shift $\Delta E_{\text {ind }}$. The latter can be obtained by the application of fourth-order perturbation theory;

$$
\Delta E_{\text {ind }}=\operatorname{Re}\left\{\sum_{t, s, r} \frac{\left\langle i\left|H_{\text {int }}\right| t\right\rangle\left\langle t\left|H_{\text {int }}\right| s\right\rangle\left\langle s\left|H_{\text {int }}\right| r\right\rangle\left\langle r\left|H_{\text {int }}\right| i\right\rangle}{\left(E_{i}-E_{t}\right)\left(E_{i}-E_{s}\right)\left(E_{i}-E_{r}\right)}\right\}
$$

where $|i\rangle$ is the unperturbed state in which both A and B are in their electronic ground state; $|r\rangle,|s\rangle$ and $|t\rangle$ are virtual states, and $E_{n}$ is the energy of state $|n\rangle$. These states can be written in the form;

$$
|n\rangle=\left|\operatorname{mol}_{n}\right\rangle\left|\operatorname{rad}_{n}\right\rangle \equiv\left|\operatorname{mol}_{n} ; \operatorname{rad}_{n}\right\rangle,
$$

with $\left|\operatorname{mol}_{n}\right\rangle$ and $\left|\operatorname{rad}_{n}\right\rangle$ defining the status of all particle and radiation states, respectively. The laser-induced interaction involves the absorption of a 'real' input photon at one molecule and the stimulated emission of a 'real' photon at the other, with one virtual photon acting as mediator between them (fig. 1), and also through the photon creation and annihilation events occurring at one molecule with coupling to the other by a virtual mediator (fig. 2). The molecules and throughput radiation suffers no overall change in state. To determine a result for $\Delta E_{\text {ind }}$ requires the insertion of equations (2) and (3) into (4). Using the fact that $\boldsymbol{\mu}(\xi)$ and $\mathbf{d}^{\perp}\left(\mathbf{R}_{\xi}\right)$ operate on $\left|\operatorname{mat}_{n}\right\rangle$ and $\left|\operatorname{rad}_{n}\right\rangle$, respectively, the latter through the relations $a^{(\lambda)}(\mathbf{p})|n(\mathbf{p}, \lambda)\rangle=\sqrt{n}|(n-1)(\mathbf{p}, \lambda)\rangle$ and $a^{\dagger(\lambda)}(\mathbf{p})|n(\mathbf{p}, \lambda)\rangle=\sqrt{n+1}|n+1(\mathbf{p}, \lambda)\rangle$, we arrive at;

$$
\begin{aligned}
\Delta E_{\text {ind }}= & \left(\frac{n \hbar c k}{\varepsilon_{0} V}\right) \operatorname{Re}\left\{e_{i}^{(1)} \bar{e}_{l}^{(2)} \alpha_{i j}^{\mathrm{A}}(k) V_{j k}(k, \mathbf{R}) \alpha_{k l}^{\mathrm{B}}(k) \exp (i \mathbf{k} \cdot \mathbf{R})\right. \\
& \left.+\frac{1}{2} e_{i}^{(1)} \bar{e}_{l}^{(2)} V_{j k}(0, \mathbf{R})\left(\beta_{i j l}^{\mathrm{A}}(k) \mu_{k}^{\mathrm{B}}+\mu_{k}^{\mathrm{A}} \beta_{i j l}^{\mathrm{B}}(k)\right)\right\}
\end{aligned}
$$

using the implied summation convention for repeated Cartesian tensor indices and with the assumption that the polarisabilities are index-symmetric. Furthermore, $\mathbf{k}$ and $\hbar c k$ denote the input wave-vector and photon energy, respectively, $V_{j k}$ signifies the fully retarded resonance electric dipole - electric dipole interaction tensor ${ }^{14}$ which is $^{1}$ dependent on the intermolecular displacement vector, $\mathbf{R} \equiv \mathbf{R}_{B}-\mathbf{R}_{A}$, also $\mu_{k}^{\xi}, \alpha_{i j}$ and $\beta_{i j k}$ are the static (permanent) dipole moment, generalised molecular polarisability and generalised hyperpolarisability, respectively. From equation (6) the laser-induced force $\mathbf{F}_{\text {ind }}=-\partial \Delta E_{\text {ind }} / \partial \mathbf{R}$ emerges as follows; 


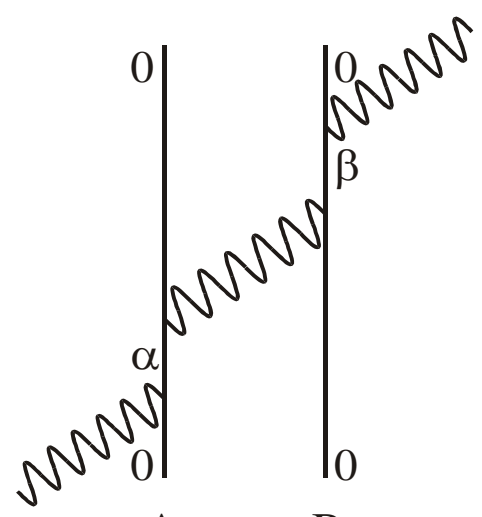

A

B

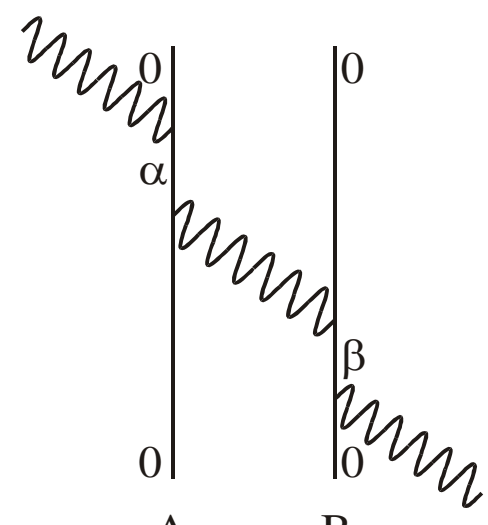

A

B

Fig. 1. Two typical Feynman diagrams (each with twenty-three further permutations) for calculation of the laser-induced interaction energy: 0 denotes the ground state level, $\alpha$ and $\beta$ are excited levels for the nanoparticles A and $\mathrm{B}$, respectively.

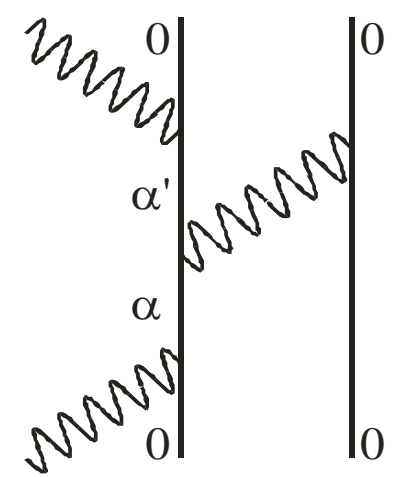

A

B

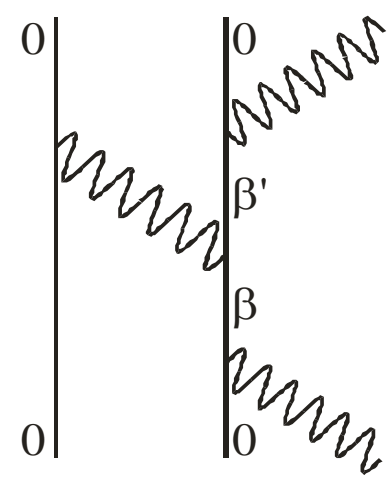

A

B

Fig. 2. Two further Feynman diagrams (each with twenty-three further permutations) for calculation of the laser-induced interaction energy: $\alpha$ and $\alpha$ ' are two excited levels for nanoparticle A, and $\beta, \beta$ ' represent excited levels for nanoparticle B.

$$
\begin{aligned}
F_{m}= & \left(\frac{I}{8 \pi \varepsilon_{0}^{2} c R^{4}}\right)\left[2 \operatorname { R e } ( e _ { i } ^ { ( 1 ) } \overline { e } _ { l } ^ { ( 2 ) } \alpha _ { i j } ^ { \mathrm { A } } ( k ) \alpha _ { k l } ^ { \mathrm { B } } ( k ) ) \left\{-k^{3} R^{3}\left(\cos k R \sin (\mathbf{k} \cdot \mathbf{R}) \hat{k}_{m}+\sin k R \cos (\mathbf{k} \cdot \mathbf{R}) \hat{R}_{m}\right)\right.\right. \\
& \times\left(\delta_{j k}-\hat{R}_{j} \hat{R}_{k}\right)-k^{2} R^{2}\left[\cos k R \cos (\mathbf{k} \cdot \mathbf{R})\left(2 \delta_{j k} \hat{R}_{m}+\delta_{m j} \hat{R}_{k}+\delta_{m k} \hat{R}_{j}-6 \hat{R}_{j} \hat{R}_{k} \hat{R}_{m}\right)-\hat{k}_{m} \sin k R\right. \\
& \left.\times \sin (\mathbf{k} \cdot \mathbf{R})\left(\delta_{j k}-3 \hat{R}_{j} \hat{R}_{k}\right)\right]+k R\left[3 \sin k R \cos (\mathbf{k} \cdot \mathbf{R})\left(\delta_{j k} \hat{R}_{m}+\delta_{m j} \hat{R}_{k}+\delta_{m k} \hat{R}_{j}-5 \hat{R}_{j} \hat{R}_{k} \hat{R}_{m}\right)+\hat{k}_{m}\right. \\
& \left.\left.\times \cos k R \sin (\mathbf{k} \cdot \mathbf{R})\left(\delta_{j k}-3 \hat{R}_{j} \hat{R}_{k}\right)\right]+3 \cos k R \cos (\mathbf{k} \cdot \mathbf{R})\left(\delta_{j k} \hat{R}_{m}+\delta_{m j} \hat{R}_{k}+\delta_{m k} \hat{R}_{j}-5 \hat{R}_{j} \hat{R}_{k} \hat{R}_{m}\right)\right\} \\
& \left.+3 \operatorname{Re}\left(e_{i}^{(1)} \bar{e}_{l}^{(2)}\left\{\beta_{i j l}^{\mathrm{A}}(k) \mu_{k}^{\mathrm{B}}(k)+\beta_{i j l}^{\mathrm{B}}(k) \mu_{k}^{\mathrm{A}}(k)\right\}\right)\left(\delta_{j k} \hat{R}_{m}+\delta_{m j} \hat{R}_{k}+\delta_{m k} \hat{R}_{j}-5 \hat{R}_{j} \hat{R}_{k} \hat{R}_{m}\right)\right]
\end{aligned}
$$

where $I=n \hbar c^{2} k / V$ is the input irradiance. On reducing equation (7) into components and setting $\mathbf{k} \cdot \mathbf{R}=0$ and $\beta_{i j l}^{\xi}=0$, the resulting expression is equivalent to the result determined classically. ${ }^{7}$ 


\section{B. Parallel SWCNTs}

The first system to be examined in detail is a pair of SWCNTs aligned in parallel along the X-axis of a system frame, both perpendicular to $\mathbf{R}$ - the latter identified with the Z-axis - and with polarized throughput radiation having its $\mathbf{e}$ vector defined by the angles $\phi$ and $\theta$ with $\mathbf{R}$ in the XZ-plane, and with the nanotube axis in the XY-plane, respectively as shown in fig. 3. Defining the whole system in terms of this Cartesian frame, employing the explicit form of the $V_{J K}$

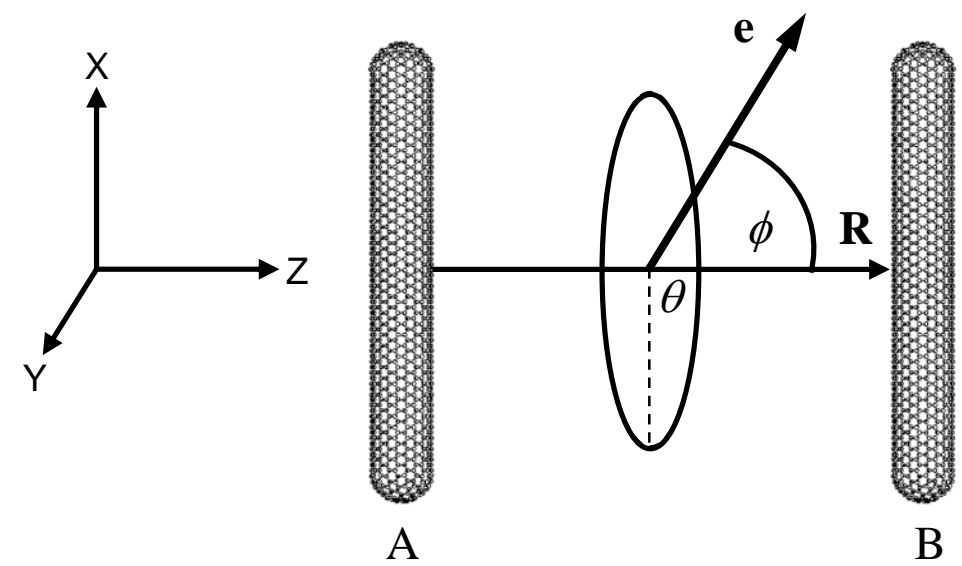

Fig. 3. Geometry of a parallel nanotube system irradiated in a fixed direction.

tensor and recognizing that ${ }^{15}$ for each nanotube $\alpha_{\mathrm{YY}}=\alpha_{\mathrm{ZZ}}=\alpha_{\perp}$ and $\alpha_{\mathrm{XX}}=\alpha_{\|}$, equation (6) becomes;

$$
\begin{aligned}
\Delta E_{\text {ind }} & =\left(\frac{I}{4 \pi \varepsilon_{0}^{2} c}\right)\left\{\left(\alpha_{\perp}^{2} \sin ^{2} \phi \sin ^{2} \theta+\alpha_{\|}^{2} \cos ^{2} \phi\right)\left(\frac{\cos k R}{R^{3}}+\frac{k \sin k R}{R^{2}}-\frac{k^{2} \cos k R}{R}\right)\right. \\
& \left.-2 \alpha_{\perp}^{2} \sin ^{2} \phi \cos ^{2} \theta\left(\frac{\cos k R}{R^{3}}+\frac{k \sin k R}{R^{2}}\right)\right\} \cos (\mathbf{k} \cdot \mathbf{R}),
\end{aligned}
$$

where the superscripts A and B are suppressed due to the chemical equivalence of the nanotube pair. Using the earlier differential expression, the laser-induced force is determined from equation (8) as;

$$
\begin{aligned}
F_{z} & =\left(\frac{I}{4 \pi \varepsilon_{0}^{2} c R^{4}}\right)\left\{( \alpha _ { \perp } ^ { 2 } \operatorname { s i n } ^ { 2 } \phi ( 1 - 3 \operatorname { c o s } ^ { 2 } \theta ) + \alpha _ { \| } ^ { 2 } \operatorname { c o s } ^ { 2 } \phi ) \left(3 \hat{R}_{z} \cos k R \cos (\mathbf{k} \cdot \mathbf{R})\right.\right. \\
& +k R\left[3 \hat{R}_{z} \sin k R \cos (\mathbf{k} \cdot \mathbf{R})+\hat{k}_{z} \cos k R \sin (\mathbf{k} \cdot \mathbf{R})\right]-k^{2} R^{2}\left[\hat{R}_{z} \cos k R \cos (\mathbf{k} \cdot \mathbf{R})\right. \\
& \left.\left.-\hat{k}_{z} \sin k R \sin (\mathbf{k} \cdot \mathbf{R})\right]\right)-\left(\alpha_{\perp}^{2} \sin ^{2} \phi \sin ^{2} \theta+\alpha_{\|}^{2} \cos ^{2} \phi\right)\left(k^{2} R^{2} \hat{R}_{z} \cos k R \cos (\mathbf{k} \cdot \mathbf{R})\right. \\
& \left.\left.+k^{3} R^{3}\left[\hat{R}_{z} \sin k R \cos (\mathbf{k} \cdot \mathbf{R})+\hat{k}_{z} \cos k R \sin (\mathbf{k} \cdot \mathbf{R})\right]\right)\right\} .
\end{aligned}
$$

The leading term of equation (9), $F_{z}^{0}$, in the short-range region ( $k R \ll 1$ ), is found by taking the leading terms in the Taylor series expansions of $\sin k R, \cos k R, \sin (\mathbf{k} \cdot \mathbf{R})$ and $\cos (\mathbf{k} \cdot \mathbf{R})$ to give;

$$
F_{z}^{0}=\left(\frac{3 I \hat{R}_{z}}{4 \pi \varepsilon_{0}^{2} c R^{4}}\right)\left(\alpha_{\perp}^{2} \sin ^{2} \phi\left(1-3 \cos ^{2} \theta\right)+\alpha_{\|}^{2} \cos ^{2} \phi\right)
$$


On the assumption that the $\alpha_{\perp}$ and $\alpha_{\|}$values are consistent with the static polarisabilities given in reference 16 , for $R=$ $2 \mathrm{~nm}$ and $I=1 \times 10^{16} \mathrm{~W} \mathrm{~m}^{-2}$, then for SWCNTs $200 \mathrm{~nm}$ in length and $0.4 \mathrm{~nm}$ in radius, equation (10) gives a force $F_{z}^{0}$ of magnitude: (i) $\sim 10 \mu N$ for $\phi=0^{\circ}$ (independent of $\theta$ ); (ii) $F_{z}^{0} \sim-1 p N$ for $\phi=90^{\circ}, \theta=0^{\circ}$; (iii) $F_{z}^{0} \sim 0.5 p N$ for $\phi=90^{\circ}, \theta=90^{\circ}$.

\section{Tumbling nanotube pair}

The second case is where the SWCNTs have arbitrary mutual orientation but the pair can freely tumble in the field of the input radiation. Here the angle between the long axis of each nanotube $\xi$ and the $\mathbf{R}$-vector (the Z-direction of the system frame) is defined as $\phi_{\xi}$ - see fig. 4. Furthermore, $\theta$ is the angle between the long axis projections of both

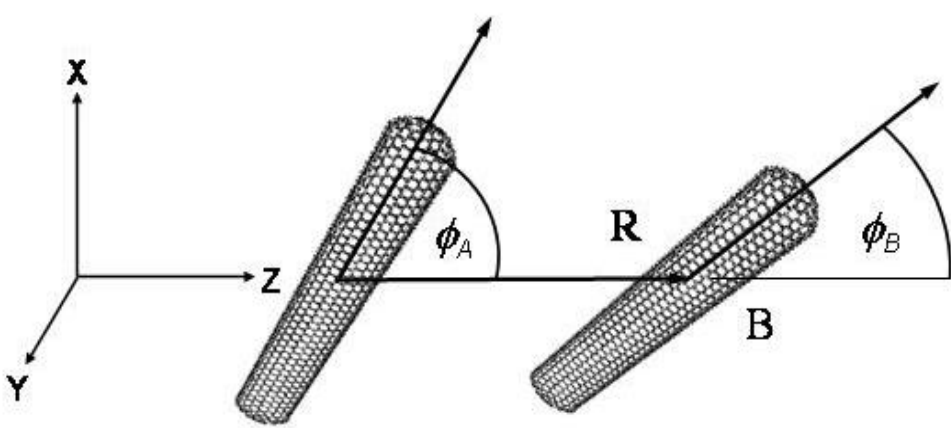

A

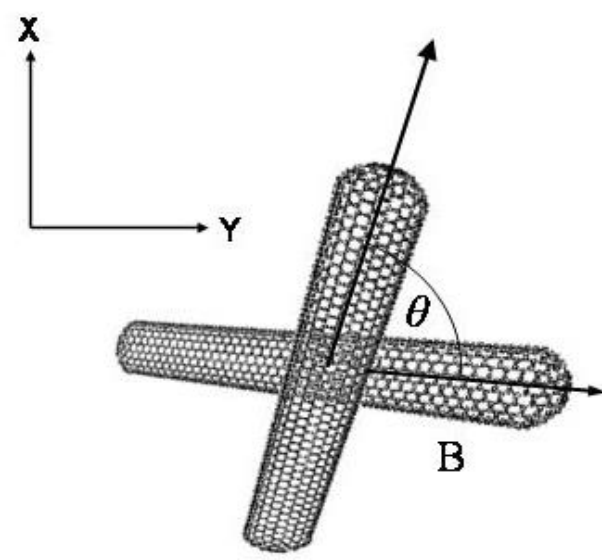

A

Fig. 4. Geometry of a nanotube pair with fixed, arbitrary mutual orientation.

nanotubes $\mathrm{A}$ and $\mathrm{B}$ on the plane perpendicular to $\mathbf{R}$, and the X-direction of the system frame is chosen such that the long axis of nanotube A resides in the XZ-plane. Assuming that the SWCNTs are isotropically averaged with respect to the incoming light a phased average $\operatorname{method}^{17}$ is required to account for the phase factor $\exp (\mathbf{i k} \cdot \mathbf{R})$ in equation $(6)-\mathrm{a}$ feature that reflects the creation and annihilation of a 'real' photon at differing positions, i.e. one at $\mathrm{A}$ and the other at $\mathrm{B}$. Hence we first obtain;

$$
\Delta E_{\text {ind }}=\left(\frac{I}{\varepsilon_{0} c}\right) \operatorname{Re}\left\{\frac{1}{3} j_{0}(k R) \alpha_{i j}^{\mathrm{A}} V_{j k} \alpha_{k i}^{\mathrm{B}}-\frac{3}{2} j_{2}(k R)\left[-\frac{1}{3} \hat{R}_{i} \alpha_{i j}^{\mathrm{A}} V_{j k} \alpha_{k l}^{\mathrm{B}} \hat{R}_{l}+\frac{1}{9} \alpha_{i j}^{\mathrm{A}} V_{j k} \alpha_{k i}^{\mathrm{B}}\right]\right\}
$$

Here $j_{n}(k R)$ are spherical Bessel functions defined by;

$$
j_{0}(k R)=\frac{\sin k R}{k R}, \quad j_{2}(k R)=\left(\frac{-1}{k R}+\frac{3}{k^{3} R^{3}}\right) \sin k R-\frac{3 \cos k R}{k^{2} R^{2}}
$$

Next, $\alpha_{i j}^{\mathrm{A}}$ and $\alpha_{k i}^{\mathrm{B}}$ are contracted with the $V_{j k}$ tensor and cast in terms of $\alpha_{\perp}$ and $\alpha_{\|}$. Finally, the laser-induced force is determined, giving the following result; 


$$
\begin{aligned}
F_{z}= & \left(\frac{I k^{3} \hat{R}_{z}}{8 \pi \varepsilon_{0}^{2} c}\right)\left\{\frac { \operatorname { c o s } ^ { 2 } k R - \operatorname { s i n } ^ { 2 } k R } { k R ^ { 2 } } \left[\left(1-\eta \cos ^{2} \phi_{A}\right)\left(\alpha_{\|}^{2} \cos ^{2} \theta\left(1-\eta \cos ^{2} \phi_{B}\right)+\alpha_{\|} \alpha_{\perp} \sin ^{2} \theta\right)+\alpha_{\|} \alpha_{\perp}\right.\right. \\
& \left.\times \sin ^{2} \theta\left(1-\eta \cos ^{2} \phi_{B}\right)+\alpha_{\perp}^{2} \cos ^{2} \theta\right]-\frac{\sin k R \cos k R}{k^{2} R^{3}}\left[6 \left(( 1 - \eta \operatorname { c o s } ^ { 2 } \phi _ { A } ) \left(\alpha_{\|}^{2} \cos ^{2} \theta\left(1-\eta \cos ^{2} \phi_{B}\right)\right.\right.\right. \\
& \left.\left.+\alpha_{\|} \alpha_{\perp} \sin ^{2} \theta\right)+\alpha_{\|} \alpha_{\perp} \sin ^{2} \theta\left(1-\eta \cos ^{2} \phi_{B}\right)+\alpha_{\perp}^{2} \cos ^{2} \theta\right)-8 \alpha_{\|}^{2} \eta^{2} \sin \phi_{A} \sin \phi_{B} \cos \phi_{A} \cos \phi_{B} \\
& \times \cos \theta]+\frac{\sin ^{2} k R-\cos ^{2} k R}{k^{3} R^{4}}\left[6 \left(\left(1-\eta \cos ^{2} \phi_{A}\right)\left(\alpha_{\|}^{2} \cos ^{2} \theta\left(1-\eta \cos ^{2} \phi_{B}\right)+\alpha_{\|} \alpha_{\perp} \sin ^{2} \theta\right)+\alpha_{\|} \alpha_{\perp}\right.\right. \\
& \left.\times \sin ^{2} \theta\left(1-\eta \cos ^{2} \phi_{B}\right)+\alpha_{\perp}^{2} \cos ^{2} \theta\right)-14 \alpha_{\|}^{2} \eta^{2} \sin \phi_{A} \sin \phi_{B} \cos \phi_{A} \cos \phi_{B} \cos \theta+4 \alpha_{\|}^{2}(1-\eta \\
& \left.\left.\times \sin ^{2} \phi_{A}\right)\left(1-\eta \sin ^{2} \phi_{B}\right)\right]+\frac{\sin k R \cos k R}{k^{4} R^{5}}\left[1 6 \left(( 1 - \eta \operatorname { c o s } ^ { 2 } \phi _ { A } ) \left(\alpha_{\|}^{2} \cos { }^{2} \theta\left(1-\eta \cos ^{2} \phi_{B}\right)+\alpha_{\|} \alpha_{\perp}\right.\right.\right. \\
& \left.\left.\times \sin ^{2} \theta\right)+\alpha_{\|} \alpha_{\perp} \sin ^{2} \theta\left(1-\eta \cos ^{2} \phi_{B}\right)+\alpha_{\perp}^{2} \cos ^{2} \theta\right)-48 \alpha_{\|}^{2} \eta^{2} \sin \phi_{A} \sin \phi_{B} \cos \phi_{A} \cos \phi_{B} \cos \theta \\
& \left.+32 \alpha_{\|}^{2}\left(1-\eta \sin ^{2} \phi_{A}\right)\left(1-\eta \sin ^{2} \phi_{B}\right)\right]+\left[\frac{\cos ^{2} k R-\sin ^{2} k R}{k^{5} R^{6}}-\frac{\sin k R \cos k R}{k^{6} R^{7}}\right]\left[6 \left(\left(1-\eta \cos \phi_{A}\right)\right.\right. \\
& \left.\times\left(\alpha_{\|}^{2} \cos ^{2} \theta\left(1-\eta \cos ^{2} \phi_{B}\right)+\alpha_{\|} \alpha_{\perp} \sin ^{2} \theta\right)+\alpha_{\|} \alpha_{\perp} \sin ^{2} \theta\left(1-\eta \cos ^{2} \phi_{B}\right)+\alpha_{\perp}^{2} \cos ^{2} \theta\right)-24 \alpha_{\|}^{2} \eta^{2} \\
& \left.\times \sin \phi_{A} \sin \phi_{B} \cos \phi_{A} \cos \phi_{B} \cos \theta+24 \alpha_{\|}^{2}\left(1-\eta \sin ^{2} \phi_{A}\right)\left(1-\eta \sin ^{2} \phi_{B}\right)\right],
\end{aligned}
$$

where $\eta=\left(\alpha_{\|}-\alpha_{\perp}\right) / \alpha_{\|}$. Again $F_{z}^{0}$, the short-range asymptote of equation (13), is found by taking the leading terms in the Taylor series expansion of $\sin k R$ and $\cos k R$, giving;

$$
\begin{aligned}
F_{z}^{0} & =\left(\frac{I \hat{R}_{z}}{8 \pi \varepsilon_{0}^{2} c R^{4}}\right)\left[4 \left(\left(1-\eta \cos ^{2} \phi_{A}\right)\left(\alpha_{\|}^{2} \cos ^{2} \theta\left(1-\eta \cos ^{2} \phi_{B}\right)+\alpha_{\|} \alpha_{\perp} \sin ^{2} \theta\right)\right.\right. \\
& \left.+\alpha_{\|} \alpha_{\perp} \sin ^{2} \theta\left(1-\eta \cos ^{2} \phi_{B}\right)+\alpha_{\perp}^{2} \cos ^{2} \theta\right)-10 \alpha_{\|}^{2} \eta^{2} \sin \phi_{A} \sin \phi_{B} \cos \phi_{A} \\
& \left.\times \cos \phi_{B} \cos \theta+4 \alpha_{\|}^{2}\left(1-\eta \sin ^{2} \phi_{A}\right)\left(1-\eta \sin ^{2} \phi_{B}\right)\right] .
\end{aligned}
$$

With the same specifications as earlier, we find that if parallel nanotubes are: (i) also parallel to $\mathbf{R}$, the force has a magnitude of $\sim 8 \mu N$, independent of $\theta$; (ii) orthogonal to $\mathbf{R}$, then at $\theta=0^{\circ}$ or $\theta=90^{\circ}$, equation (14) gives $\sim 8 \mu N$ or $\sim 3 n N$, respectively.

\section{OPTICAL VORTEX CONDITIONS}

\section{A. General result}

In this section attention is focused on the laser-induced interaction associated with an optical vortex, due to irradiation of the A-B pair with a Laguerre-Gaussian (LG) or other 'twisted' optical beam. Under these conditions, it is interesting to determine an expression for the torque between the nanoparticles; a means of achieving this is by again finding $\Delta E_{\text {ind }}$. The latter can be accomplished by defining a system with the positions of $\mathrm{A}$ and $\mathrm{B}$ set in cylindrical coordinates and, for simplicity, fixing the distance of the nanoparticles from the beam centre, $r$, confining them to a particular $\zeta$ value - the Greek letter denoting the radiation frame and the propagation direction of the LG beam ( $-Y$ in the Cartesian frame) - and permitting the azimuthal displacement angle, $\Delta \varphi=\varphi_{B}-\varphi_{A}$, to vary (fig. 5). Following the same procedure as in section 2 , the fourth-order perturbation method is applied to give; 


$$
\begin{aligned}
\Delta E_{\text {ind }} & =\left(\frac{I f_{l p}^{2}(r)}{\varepsilon_{0} c A_{l p}}\right) \operatorname{Re}\left\{\varepsilon_{i}^{(1)} \bar{\varepsilon}_{l}^{(2)} \alpha_{i j}^{\mathrm{A}}(k) V_{j k}(k, \mathbf{R}) \alpha_{k l}^{\mathrm{B}}(k) \exp (l \Delta \varphi)\right. \\
& \left.+\frac{1}{2} \varepsilon_{i}^{(1)} \bar{\varepsilon}_{l}^{(2)} V_{j k}(0, \mathbf{R})\left(\beta_{i j l}^{\mathrm{A}}(k) \mu_{k}^{\mathrm{B}}+\mu_{k}^{\mathrm{A}} \beta_{i j l}^{\mathrm{B}}(k)\right)\right\} .
\end{aligned}
$$

Here, $p$ accounts for all possible modes with the same angular momentum $l, A_{l p}$ are normalisation constants and $f_{l p}(r)$ is given by;

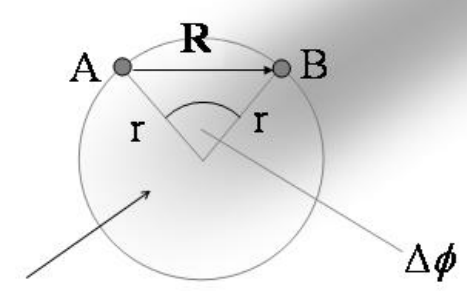

Fig. 5. Geometry of nanoparticle pair in a Laguerre-Gaussian beam.

$$
f_{l p}(r)=\frac{C_{p}^{|l|}}{\omega_{0}}\left[\frac{\sqrt{2} r}{\omega_{0}}\right]^{|l|} \exp \left(\frac{-r^{2}}{\omega_{0}^{2}}\right) L_{p}^{\mid l}\left(\frac{2 r^{2}}{\omega_{0}^{2}}\right),
$$

where $C_{p}^{|l|}$ is a normalisation constant, $\omega_{0}$ defines the Gaussian beam-waist at $z=0$ and $L_{p}^{|l|}$ is the generalised Laguerre polynomial. From (15) the laser-induced torque $\tau=\left(-\partial \Delta E_{\text {ind }} / \partial \Delta \varphi\right) \hat{\mathbf{R}}$ emerges as;

$$
\begin{aligned}
\tau & =\left(\frac{I k^{3} f_{l p}^{2} \hat{\mathbf{R}}}{8 \pi \varepsilon_{0}^{2} c A_{l p}}\right)\left[2 \operatorname { R e } ( \varepsilon _ { i } ^ { ( 1 ) } \overline { \varepsilon } _ { l } ^ { ( 2 ) } \alpha _ { i j } ^ { A } \alpha _ { k l } ^ { B } ) \left\{-\frac{\left(\delta_{j k}-\hat{R}_{j} \hat{R}_{k}\right) \sin \left(\sqrt{2} k r(1-\cos \Delta \varphi)^{1 / 2}\right) \sin \Delta \varphi}{2(1-\cos \Delta \varphi)}\right.\right. \\
& \times \cos (l \Delta \varphi)-\frac{\cos \left(\sqrt{2} k r(1-\cos \Delta \varphi)^{1 / 2}\right)}{\sqrt{2} k r(1-\cos \Delta \varphi)^{3 / 2}}\left(\left(\delta_{j k}-\hat{R}_{j} \hat{R}_{k}\right) l \sin (l \Delta \varphi)(1-\cos \Delta \varphi)\right. \\
& \left.+\left(\delta_{j k}-2 \hat{R}_{j} \hat{R}_{k}\right) \sin \Delta \varphi \cos (l \Delta \varphi)\right)+\left(\frac{\left(\delta_{j k}-3 \hat{R}_{j} \hat{R}_{k}\right) \sin \left(\sqrt{2} k r(1-\cos \Delta \varphi)^{1 / 2}\right)}{2 k^{2} r^{2}(1-\cos \Delta \varphi)^{2}}\right. \\
& \left.+\frac{\left(\delta_{j k}-3 \hat{R}_{j} \hat{R}_{k}\right) \cos \left(\sqrt{2} k r(1-\cos \Delta \varphi)^{1 / 2}\right)}{2 \sqrt{2} k^{3} r^{3}(1-\cos \Delta \varphi)^{5 / 2}}\right)\left(l \sin (l \Delta \varphi)(1-\cos \Delta \varphi)+\frac{3}{2} \sin \Delta \varphi\right. \\
& \times \cos (l \Delta \varphi))\}+3 \operatorname{Re}\left(\varepsilon_{i}^{(1)} \bar{\varepsilon}_{l}^{(2)}\left[\beta_{i j l}^{\mathrm{A}} \mu_{k}^{\mathrm{B}}+\beta_{i j l}^{\mathrm{B}} \mu_{k}^{\mathrm{A}}\right]\right) \frac{\left(\delta_{j k}-3 \hat{R}_{j} \hat{R}_{k}\right) \sin \Delta \varphi}{\left.2 \sqrt{2} r^{3}(1-\cos \Delta \varphi)^{5 / 2}\right],}
\end{aligned}
$$

using $R=\sqrt{2} r(1-\cos \Delta \varphi)^{1 / 2}$ by the cosine rule and, here and henceforth, suppressing the $f_{l p}$ and $\alpha_{i j}$ dependence. 


\section{B. Spherical nanoparticles}

A system that is interesting to examine in detail is based on the scheme defined by section 2B and illustrated by fig. 3, but with the nanotubes replaced by a spherical nanoparticle pair. Under an irradiating LG beam, the laser-induced energy shift for this system is determined by expressing the polarisation unit vector in simplified cylindrical coordinates, i.e. as $\theta=0$ due to the fixed direction of the LG beam, and defining the system in terms of the Cartesian frame. Hence, in cognisance of the fact $\mu_{k}^{\xi}=0$ for spherical particles, equation (15) becomes;

$$
\begin{aligned}
\Delta E_{\text {ind }}= & \left(\frac{I f_{l p}^{2}}{\varepsilon_{0} c A_{l p}}\right) \operatorname{Re}\left\{\sum_{J, K} \sin ^{2} \phi \cdot \alpha_{Z J}^{\mathrm{A}} V_{J K} \alpha_{K Z}^{\mathrm{B}}-\sin \phi \cos \phi \cdot \alpha_{Z J}^{\mathrm{A}} V_{J K} \alpha_{K X}^{\mathrm{B}}\right. \\
& \left.-\sin \phi \cos \phi \cdot \alpha_{X J}^{\mathrm{A}} V_{J K} \alpha_{K Z}^{\mathrm{B}}+\cos ^{2} \phi \cdot \alpha_{X J}^{\mathrm{A}} V_{J K} \alpha_{K X}^{\mathrm{B}}\right\} \cos (l \Delta \varphi) .
\end{aligned}
$$

Taking the explicit form of the $V_{J K}$ tensor and recognizing that only $\alpha_{\mathrm{xx}}=\alpha_{\mathrm{ZZ}}=\alpha$ exist for a spherical pair, equation (18) is rewritten as;

$$
\begin{aligned}
\Delta E_{\mathrm{ind}}= & \left(\frac{I f_{l p}^{2} \alpha^{2}}{4 \pi \varepsilon_{0}^{2} c A_{l p}}\right)\left\{\cos ^{2} \phi\left(\frac{\cos k R}{R^{3}}+\frac{k \sin k R}{R^{2}}-\frac{k^{2} \cos k R}{R}\right)\right. \\
& \left.-2 \sin ^{2} \phi\left(\frac{\cos k R}{R^{3}}+\frac{k \sin k R}{R^{2}}\right)\right\} \cos (l \Delta \varphi) .
\end{aligned}
$$

The laser-induced torque is again determined from the differential expression of the previous sub-section to give;

$$
\begin{aligned}
\tau & =\left(\frac{I k^{3} f_{l p}^{2} \alpha^{2} \hat{\mathbf{R}}}{4 \pi \varepsilon_{0}^{2} c A_{l p}}\right)\left\{-\frac{\sin \left(\sqrt{2} k r(1-\cos \Delta \varphi)^{1 / 2}\right)}{2(1-\cos \Delta \varphi)} \cos ^{2} \phi \sin \Delta \varphi \cos (l \Delta \varphi)-\frac{\cos \left(\sqrt{2} k r(1-\cos \Delta \varphi)^{1 / 2}\right)}{2 \sqrt{2} k r(1-\cos \Delta \varphi)^{3 / 2}}\right. \\
& \times\left(2 l \cos ^{2} \phi \sin (l \Delta \varphi)(1-\cos \Delta \varphi)+\left(\left(1-3 \sin ^{2} \phi\right)+\cos ^{2} \phi\right) \sin \Delta \varphi \cos (l \Delta \varphi)\right)\left(\left(1-3 \sin ^{2} \phi\right)\right. \\
& \left.\times \frac{\sin \left(\sqrt{2} k r(1-\cos \Delta \varphi)^{1 / 2}\right)}{2 k^{2} r^{2}(1-\cos \Delta \varphi)^{2}}+\frac{\left(1-3 \sin ^{2} \phi\right) \cos \left(\sqrt{2} k r(1-\cos \Delta \varphi)^{1 / 2}\right)}{2 \sqrt{2} k^{3} r^{3}(1-\cos \Delta \varphi)^{5 / 2}}\right)(l \sin (l \Delta \varphi)(1-\cos \Delta \varphi) \\
& \left.\left.+\frac{3}{2} \sin \Delta \varphi \cos (l \Delta \varphi)\right)\right\} .
\end{aligned}
$$

In the short-range region $(k r \ll 1)$ equation $(20)$ becomes;

$$
\begin{aligned}
\tau^{0}= & \frac{I f_{l p}^{2} \alpha^{2}\left(1-3 \sin ^{2} \phi\right) \cos \left(\sqrt{2} k r(1-\cos \Delta \varphi)^{1 / 2}\right) \hat{\mathbf{R}}}{8 \sqrt{2} \pi \varepsilon_{0}^{2} r^{3} c A_{l p}(1-\cos \Delta \varphi)^{5 / 2}}(l \sin (l \Delta \varphi)(1-\cos \Delta \varphi) \\
& \left.\left.+\frac{3}{2} \sin \Delta \varphi \cos (l \Delta \varphi)\right)\right] .
\end{aligned}
$$

By inspection of (20) and (21) it is determined that an additional contribution to the torque will be observed for an irradiating LG beam $(l \neq 0)$ in comparison to conventional Gaussian laser light $(l=0)$, for example, due to the presence of the first term of (21). 


\section{DISCUSSION}

When irradiated by intense laser light, dielectric nanoparticles experience distinctive inter-particle forces and torques that prove to scale linearly with the applied irradiance. By a quantum electrodynamical analysis we have identified the detailed form of these forces and shown that they originate in pairwise processes of stimulated scattering. Calculations have been performed for a pair of carbon nanotubes and the results, based on static polarizability data, give indicative values. It has been shown that these forces can be either positive or negative, according to conditions; the descriptor 'binding force' found in various papers ${ }^{7,8}$ is evidently an over-simplification of the physics and could be misleading. Further highly distinctive optomechanical features have been identified in specific connection with nanoparticles within an optical vortex (a 'twisted' beam such as a Laguerre-Gaussian mode). It has been demonstrated that these additional contributions can strongly dominate the torque exerted between nanoparticles. It is as yet unknown how such effects may be manifest in collective molecular motion or property modifications, but preliminary studies suggest such features such as optical ordering, clustering and trapping. These are issues that are currently the subject of further research in the group.

\section{ACKNOWLEDGMENT}

DSB is funded by a studentship from the Engineering and Physical Sciences Research Council.

\section{REFERENCES}

1. A. Ashkin, “Optical trapping and manipulation of neutral particles using lasers”, Proc. Natl. Acad. Sci. USA 94, pp. 4853-4860, 1997.

2. J. E. Molloy, K. Dholakia and M. J. Padgett, “Optical tweezers in a new light”, J. Mod. Opt. 50, pp. 1501-1507, 2003.

3. K. Svoboda and S. M. Block, "Biological applications of optical forces", Annu. Rev. Biophys. Biomol. Struct. 23, pp. 247-285, 1994.

4. V. I. Balykin, V. G. Minogin and V. S. Letokhov, "Electromagnetic trapping of cold atoms", Rep. Prog. Phys. 63, pp. 1429-1510, 2000.

5. D. G. Grier, “A revolution in optical manipulation”, Nature 424, pp. 810-816, 2003.

6. M. M. Burns, J.-M. Fournier and J. A. Golovchenko, “Optical binding”, Phys. Rev. Lett. 63, pp. 1233-1236, 1989.

7. F. Depasse and J.-M. Vigoureux, "Optical binding force between two Rayleigh particles", J. Phys. D: Appl. Phys. 27, pp. 914-919, 1994.

8. P. C. Chaumet and M. Nieto-Vesperinas, "Optical binding of particles with or without the presence of a flat dielectric surface”, Phys. Rev. B 64, 035422, 2001.

9. S. K. Mohanty, J. T. Andrews and P. K. Gupta, “Optical binding between dielectric particles”, Opt. Express 12, pp. 2749-2756, 2004.

10. D. L. Andrews and D. S. Bradshaw, "Laser-induced forces between carbon nanotubes”, Opt. Lett., in press.

11. G. Cao, Nanostructures and Nanomaterials: Synthesis, Properties and Applications (Imperial College Press, London, 2004), p. 238.

12. L. C. Dávila Romero, D. L. Andrews and M. Babiker, "A quantum electrodynamics framework for the nonlinear optics of twisted beams”, J. Opt. B: Quantum Semiclass. Opt. 4, pp. S66-S72, 2002.

13. D. P. Craig and T. Thirunamachandran, Molecular Quantum Electrodynamics (Dover, Mineola, New York, 1998).

14. G. J. Daniels, R. D. Jenkins, D. S. Bradshaw and D. L. Andrews, "Resonance energy transfer: The unified theory revisited", J. Chem. Phys. 119, pp. 2264-2274, 2003.

15. C. G. Gray and K. E. Gubbins, Theory of Molecular Fluids (Clarendon Press, Oxford, 1984) p. 545.

16. G. Y. Guo, K. C. Chu, D.-S. Wang and C.-G. Duan, "Static polarizability of carbon nanotubes: ab initio independent-particle calculations", Comput. Mater. Sci. 30, pp. 269-273, 2004.

17. D. L. Andrews and M. J. Harlow, "Phased and Boltzmann-weighted rotational averages", Phys. Rev. A 29, pp. 2796-2806, 1984.

*david.andrews@physics.org 